Annales Academiæ Scientiarum Fennicæ

Series A. I. Mathematica

Volumen 14, 1989, 129-131

\title{
ON THE INFINITY OF THE LONERGAN-HOSACK PRESENTATION
}

\author{
Markku Niemenmaa and Gerhard Rosenberger
}

\section{Introduction}

In 1984 F.D. Lonergan and J. Hosack [3] introduced the following group presentation:

$$
G=\left\langle x, z: z^{3} x z^{3} x^{-1}=z^{5} x^{2} z^{2} x^{2}=1\right\rangle .
$$

According to the authors there was good reason to believe that the presentation is that of a finite group. However, they reported that attempts to prove finiteness by using the Todd-Coxeter algorithm were unsuccessful.

By using the computer algebra system CAYLEY M. Slattery [5] managed to show in 1985 that $G$ is infinite. To be precise, Slattery showed that $G$ has a factor group which is infinite.

In this short note we wish to point out that a lot more can be said about the structure of $G$. In fact, we shall consider the presentation

$$
G(n)=\left\langle x, z: z^{n} x z^{n} x^{-1}=z^{n+2} x^{2} z^{2} x^{2}=1\right\rangle
$$

with $n \geq 2$. In particular, our results hold for $G=G(3)$. For the background material of this note the reader is advised to consult [7].

\section{Main theorem}

Consider the presentation $G(n)$ given in the introduction with $n \geq 1$. If $n=1$, then $z x^{2}=x^{2} z$ and $z^{5}=z^{-5}=x^{4}$. Consequently $G(1)$ is a finite group of order 40 . Now we shall establish the

Theorem. Let $n \geq 2$ and consider the presentation $G(n)$. Now

(1) $G(n)$ is a nontrivial free product with amalgamation,

(2) $G(n)$ has a subgroup of finite index mapping onto a free group of rank 2 and $G(n)$ has a free subgroup of rank 2 ,

(3) $G(n)$ has a generating pair $\{u, v\}$ such that the subgroup $\left\langle u^{k}, v^{k}\right\rangle$ is free of rank 2 for a sufficiently large integer $k$,

(4) $G(n)$ is SQ-universal (i.e. every countable group is embeddable in some factor group of $G(n))$. 
Proof. (1) If $n$ is even, then the free product $Z_{4} * Z_{2}=\left\langle x, z: x^{4}=z^{2}=1\right\rangle$ is an epimorphic image of $G(n)$. If $n$ is odd (then $n \geq 3$ ), we have $D=\langle x, z$ : $\left.z^{n}=\left(z^{2} x^{2}\right)^{2}=1\right\rangle$ as an epimorphic image of $G(n)$. Now $D$ is a nontrivial free product of $H_{1}$ and $H_{2}$ with the amalgamated subgroup $H$, where $H_{1}=\langle x\rangle \cong Z$, $H_{2}=\left\langle y, z: z^{n}=\left(z^{2} y\right)^{2}=1\right\rangle$ and $H=\left\langle x^{2}\right\rangle \cong\langle y\rangle$. By Lemma 3.2 of [6] we conclude that $G(n)$ is a nontrivial free product with amalgamation for every $n \geq 2$.

(2) The free product $Z_{4} * Z_{2}$ has the triangle-group

$$
T(2,4,5)=\left\langle a, b: a^{2}=b^{4}=(a b)^{5}=1\right\rangle
$$

as an epimorphic image and clearly $G(n)$ has $T(2,4,5)$ as an epimorphic image provided $n$ is even. Next consider $D$ (the free product with amalgamation) introduced in the first part of the proof. Now $D$ has the triangle-group

$$
T(n, 7,2)=\left\langle a, b: a^{n}=b^{7}=(a b)^{2}=1\right\rangle
$$

as an epimorphic image and naturally $G(n)$ has $T(n, 7,2)$ as an epimorphic image for odd $n(n \geq 3)$.

Thus $G(n)$ has as an epimorphic image a triangle-group

$$
T(p, q, r)=\left\langle a, b: a^{p}=b^{q}=(a b)^{r}=1\right\rangle
$$

with $2 \leq p, q, r$ and $(1 / p)+(1 / q)+(1 / r)<1$. Now $T(p, q, r)$ has a surface group $F(g)$ of genus $g \geq 2$ as a subgroup of finite index (see [7]). Since $F(g)(g \geq 2)$ has a free group of rank 2 as an epimorphic image, it follows by considering the pre-image of $F(g)$ in $G(n)$ that $G(n)$ has a subgroup of finite index which maps onto a free group of rank 2 and consequently $G(n)$ has a free subgroup of rank 2 .

(3) Since $T(p, q, r)$ can be regarded as a subgroup of $P S L(2, R)$ (see [7]), our assertion follows from [4].

(4) By [1] we know that a free group of rank 2 is SQ-universal. Clearly, a pre-image of an SQ-universal group is SQ-universal. Finally, by [2] it follows that a group which has an SQ-universal subgroup of finite index is also SQ-universal. The proof is complete.

Remarks. As indicated in the beginning of this paper $G(1)$ is a finite group of order 40 and consequently it has elements of finite order $\geq 2$. If we consider $G(2)$, then $z^{2} x z^{2} x^{-1}=z^{4} x^{2} z^{2} x^{2}=1$ implies $x^{2} z^{2}=z^{2} x^{2}$. Furthermore, $z^{6}=x^{-4}$ and $z^{-6}=x^{-4}$, hence $z^{12}=1$ and $x^{8}=1$. Now we state

Problem 1. Is it true that $G(n)$ has elements of finite order $\geq 2$ for $n \geq 3$ ? We also state

Problem 2. Is it true that $G(n)$ has a torsion-free normal subgroup of finite index for $n \geq 2$ ? 


\section{References}

[1] Higman, G., B.H. Neumann, and H. Neumann: Embedding theorems for groups. - J. London Math. Soc. 24, 1949, 247-254.

[2] Neumann, P.M.: The SQ-universality of some finitely generated groups. - J. Austral. Math. Soc. 16, 1973, 1-6.

[3] Lonergan, F.D., and J. Hosack: Problem 308.- Notices Amer. Math. Soc. 31, 5, 1984.

[4] Rosenberger, G.: On discrete free subgroups of linear groups.- J. London Math. Soc. (2) $17,1978,79-85$.

[5] Slattery, M.: Solution to AMS Notices query No. 308. - Cayley Bulletin 2, 1985, 37-39.

[6] Zieschang, H.: On decompositions of discontinuous groups of the plane. - Math. Z. 151, 1976, 165-188.

[7] Zieschang, H., E. Vogt, and H.D. Coldewey: Surfaces and planar discontinuous groups. - Lecture Notes in Mathematics 835. Springer-Verlag, Berlin-HeidelbergNew York, 1980.

University of Oulu

Department of Mathematics

SF-90570 Oulu

Finland

Received 8 February 1988
University of Dortmund

Department of Mathematics

D-4600 Dortmund 50

Federal Republic of Germany 